\title{
A characterization of the duality mapping for convex bodies
}

\author{
Károly J. Böröczky and Rolf Schneider
}

\begin{abstract}
We characterize the duality of convex bodies in $d$-dimensional Euclidean vector space, viewed as a mapping from the space of convex bodies containing the origin in the interior into the same space. The question for such a characterization was posed by Vitali Milman. Sufficient for a characterization, up to a trivial exception and the composition with a linear transformation, is the property that the duality interchanges pairwise intersections and convex hulls of unions.
\end{abstract}

MSC 2000: 52A20

Key words: duality of convex bodies, polar convex body, lattice endomorphism

\section{Introduction}

By $\mathbb{R}^{d}$ we denote the $d$-dimensional real Euclidean vector space, equipped with its standard scalar product $\langle\cdot, \cdot\rangle$. We assume throughout that $d \geq 2$. The set of convex bodies (compact convex subsets) in $\mathbb{R}^{d}$ which contain 0 is denoted by $\mathcal{K}_{0}^{d}$, and the subset of bodies containing 0 in the interior by $\mathcal{K}_{(0)}^{d}$. For $K \in \mathcal{K}_{(0)}^{d}$, the dual or polar body is defined by

$$
K^{*}:=\left\{x \in \mathbb{R}^{d}:\langle x, y\rangle \leq 1 \text { for all } y \in K\right\} .
$$

It is again in $\mathcal{K}_{(0)}^{d}$. The duality mapping $K \mapsto K^{*}$ has a number of remarkable properties, of which we list the following (see, for example, [4, Sect. 1.6]); they are valid for all $K, L \in \mathcal{K}_{(0)}^{d}$.

(D1) $\left(K^{*}\right)^{*}=K$.

(D2) $K \subset L$ implies $K^{*} \supset L^{*}$.

(D3) $(K \cap L)^{*}=\operatorname{conv}\left(K^{*} \cup L^{*}\right)$.

(D4) $[\operatorname{conv}(K \cup L)]^{*}=K^{*} \cap L^{*}$.

(D5) If $K \cup L$ is convex, then $(K \cap L)^{*}=K^{*} \cup L^{*}$ and $(K \cup L)^{*}=K^{*} \cap L^{*}$.

This work was supported by the EU-project DiscConvGeo (MTKD-CT-2005-014333). The first author was supported by OTKA grant 049301. 
(D6) Continuity with respect to the Hausdorff metric.

(D7) If $g \in G L(d)$, then $(g K)^{*}=g^{-t} K^{*}$.

Of course, the listed properties of a mapping $K \mapsto K^{*}$ are not independent. For example, (D3) implies (D2); (D1) and (D3) imply (D4) and (D5).

The question, which properties of the duality mapping are sufficient to characterize it, has been posed to the authors by Vitali Milman. A complete characterization would definitely require additional assumptions, since also the map $K \mapsto-K^{*}$ satisfies (D1) - (D7). Note also that a constant map $K \mapsto B$ (with $B \in \mathcal{K}_{(0)}^{d}$ fixed), as well as a map $K \mapsto g K^{*}$ (with $g \in G L(d)$ fixed), satisfies satisfies (D2) - (D6). On the other hand, when we are prepared to accept such modifications, then characterizations by a few of the above properties are possible. Here, the following result will be proved.

We write $A \vee B:=\operatorname{conv}(A \cup B)$ for $A, B \subset \mathbb{R}^{d}$ and $x_{1} \vee \cdots \vee x_{k}:=\operatorname{conv}\left\{x_{1}, \ldots, x_{k}\right\}$ for $x_{1}, \ldots, x_{k} \in \mathbb{R}^{d}$.

Theorem. Let $\psi: \mathcal{K}_{(0)}^{d} \rightarrow \mathcal{K}_{(0)}^{d}$ be a mapping satisfying

$$
\begin{aligned}
& \psi(K \cap L)=\psi(K) \vee \psi(L), \\
& \psi(K \vee L)=\psi(K) \cap \psi(L)
\end{aligned}
$$

for all $K, L \in \mathcal{K}_{(0)}^{d}$. Then either $\psi$ is constant, or there exists a linear transformation $g \in G L(d)$ such that $\psi(K)=g K^{*}$ for all $K \in \mathcal{K}_{(0)}^{d}$.

Note, in particular, that no continuity assumption is required.

If $\psi$ satisfies (1) and (2), and if we define

$$
\psi^{*}(K):=\psi(K)^{*} \quad \text { for } K \in \mathcal{K}_{(0)}^{d},
$$

then the mapping $\psi^{*}: \mathcal{K}_{(0)}^{d} \rightarrow \mathcal{K}_{(0)}^{d}$ satisfies

$$
\psi^{*}(K \cap L)=\psi^{*}(K) \cap \psi^{*}(L), \quad \psi^{*}(K \vee L)=\psi^{*}(K) \vee \psi^{*}(L) .
$$

Thus, $\psi^{*}$ is an endomorphism of the lattice $\left(\mathcal{K}_{(0)}^{d}, \cap, \vee\right)$. Gruber [1] has explicitly determined all endomorphisms of the lattice $\left(\mathcal{K}^{d}, \cap, \vee\right)$, where $\mathcal{K}^{d}$ is the system of all compact convex subsets of $\mathbb{R}^{d}$ (including the empty set). In [2], Gruber has also completely classified the endomorphisms of the lattice $\left(\mathcal{B}^{d}, \cap, \vee\right)$, where $\mathcal{B}^{d}$ is the system of all unit balls of norms on $\mathbb{R}^{d}$, that is, of all convex bodies having 0 as interior point and center of symmetry. From the latter result, one obtains the version of the theorem above where $\mathcal{K}_{(0)}^{d}$ is replaced by $\mathcal{B}^{d}$. The proof in [2] uses the central symmetry of the images in several crucial ways, therefore the general case requires a different proof.

Corollary. Let $\psi: \mathcal{K}_{(0)}^{d} \rightarrow \mathcal{K}_{(0)}^{d}$ be a mapping satisfying

$$
\begin{aligned}
\psi(\psi(K)) & =K, \\
\psi(K \cap L) & =\psi(K) \vee \psi(L)
\end{aligned}
$$


for all $K, L \in \mathcal{K}_{(0)}^{d}$. Then there exists a selfadjoint linear transformation $g \in G L(d)$ such that $\psi(K)=g K^{*}$ for all $K \in \mathcal{K}_{(0)}^{d}$.

In fact, (5) and (6) give

$$
\psi(\psi(K) \cap \psi(L))=\psi(\psi(K)) \vee \psi(\psi(L))=K \vee L,
$$

hence

$$
\psi(K \vee L)=\psi(K) \cap \psi(L)
$$

Thus, the theorem can be applied. Condition (5) excludes a constant map and forces the linear map appearing in the theorem to be selfadjoint.

The classification can be further narrowed down if (D7) is added to the assumptions of the theorem. If the map in the theorem additionally satisfies $\psi(h K)=h^{-t} \psi(K)$ for all $K \in \mathcal{K}_{(0)}^{d}$ and all $h \in G L(d)$, then there exists a real number $c$ such that $\psi(K)=c K^{*}$ for all $K \in \mathcal{K}_{(0)}^{d}$. Also a mapping $\psi$ satisfying (D5) and (D7) must be of this form, as can be shown with the methods of [3]. This was kindly pointed out to us by Monika Ludwig. We gratefully acknowledge the helpful conversations with her. We also thank Peter Gruber for useful hints.

\section{Proof of the theorem}

Suppose $\psi: \mathcal{K}_{(0)}^{d} \rightarrow \mathcal{K}_{(0)}^{d}$ is a mapping satisfying (1) and (2), and define $\psi^{*}$ by (3). It is important to notice that, by (4), $\psi^{*}$ is inclusion preserving, that is, $K \subset L$ implies $\psi^{*}(K) \subset \psi^{*}(L)$.

Our first aim is to define a mapping $\varphi$ with similar properties which is defined on the set $\mathcal{K}_{0}^{d}$ of convex bodies containing the origin, but not necessarily in the interior. Let $K \in \mathcal{K}_{0}^{d}$ be given. We choose a sequence $\left(K_{i}\right)_{i \in \mathbb{N}}$ in $\mathcal{K}_{(0)}^{d}$ with $K \in \operatorname{int} K_{i}$ for all $i$ and with $K_{i} \downarrow K$, that is, satisfying $K_{i+1} \subset K_{i}$ for all $i$ and $K=\bigcap_{i \in \mathbb{N}} K_{i}$. Put

$$
\varphi(K):=\bigcap_{i \in \mathbb{N}} \psi^{*}\left(K_{i}\right)
$$

It is easy to see (using a compactness argument) that this definition is independent of the choice of the sequence $\left(K_{i}\right)_{i \in \mathbb{N}}$. Clearly $\varphi(K) \in \mathcal{K}_{0}^{d}$. We show that properties (4) carry over to $\varphi$, for $K, L \in \mathcal{K}_{0}^{d}$. Let $K, L \in \mathcal{K}_{0}^{d}$ be given, and choose decreasing sequences $\left(K_{i}\right)_{i \in \mathbb{N}},\left(L_{i}\right)_{i \in \mathbb{N}}$ in $\mathcal{K}_{(0)}^{d}$ with $K \in \operatorname{int} K_{i}, L \in \operatorname{int} L_{i}, K=\bigcap_{i \in \mathbb{N}} K_{i}, L=$ $\bigcap_{i \in \mathbb{N}} L_{i}$. Then $K \cap L \subset \operatorname{int}\left(K_{i} \cap L_{i}\right), K_{i} \cap L_{i} \downarrow K \cap L$, and hence $\varphi(K \cap L)=$ $\bigcap_{i} \psi^{*}\left(K_{i} \cap L_{i}\right)=\bigcap_{i} \psi^{*}\left(K_{i}\right) \cap \bigcap_{i} \psi^{*}\left(L_{i}\right)=\varphi(K) \cap \varphi(L)$. Clearly, $K \vee L \subset \bigcap_{i}\left(K_{i} \vee L_{i}\right)$. If $x \in \mathbb{R}^{d} \backslash(K \vee L)$, there exists an open halfspace $H$ containing $K \vee L$ and not containing $x$. For all sufficiently large $i$ we have $K_{i} \subset H$ and $L_{i} \subset H$, hence $K_{i} \vee L_{i} \subset H$. This shows that $x \notin \bigcap_{i}\left(K_{i} \vee L_{i}\right)$ and hence that

$$
K \vee L=\bigcap_{i}\left(K_{i} \vee L_{i}\right)
$$


By definition then, and applying (7) with $K_{i}, L_{i}$ replaced by $\psi^{*}\left(K_{i}\right), \psi^{*}\left(L_{i}\right)$, we obtain

$$
\begin{aligned}
\varphi(K \vee L) & =\bigcap_{i} \psi^{*}\left(K_{i} \vee L_{i}\right)=\bigcap_{i}\left[\psi^{*}\left(K_{i}\right) \vee \psi^{*}\left(L_{i}\right)\right] \\
& =\left(\bigcap_{i} \psi^{*}\left(K_{i}\right)\right) \vee\left(\bigcap_{i} \psi^{*}\left(L_{i}\right)\right)=\varphi(K) \vee \varphi(L) .
\end{aligned}
$$

We have shown that the mapping $\varphi$ is an endomorphism of the lattice $\left(\mathcal{K}_{0}^{d}, \cap, \vee\right)$. Of course, $K \subset L$ with $K, L \in \mathcal{K}_{0}^{d}$ implies $\varphi(K) \subset \varphi(L)$. As mentioned, Gruber [1] has determined all endomorphisms of the lattice $\left(\mathcal{K}^{d}, \cap, \vee\right)$. We took Gruber's proof as a model for obtaining a classification of all endomorphisms of the lattice $\left(\mathcal{K}_{0}^{d}, \cap, \vee\right)$.

For $x \in \mathbb{R}^{d}$, we write $\bar{x}$ for the closed segment $x \vee 0$ with endpoints $x$ and 0 ; in particular, $\overline{0}=\{0\}$. If one tries to adapt Gruber's [1] approach from $\mathcal{K}^{d}$ to $\mathcal{K}_{0}^{d}$, the first thing to do is to replace the empty set $\emptyset$ by $\overline{0}$ and any point $x \in \mathbb{R}^{d}$ by the segment $\bar{x}$. In pursuing this for the cases 2.1 to 2.6 considered by Gruber, we found a shorter proof for his cases 2.4 and 2.5, and we present this here for our case of the lattice $\left(\mathcal{K}_{0}^{d}, \cap, \vee\right)$. This is still more complicated than the elegant argument used by Gruber in [2] (to prove the implication $(8) \Rightarrow(13)$ in [2]), but the latter depends on the fact that the images, being 0 -symmetric, contain 0 in their relative interiors. The final case 2.6 considered by Gruber [1] also needs a different and more elaborate argument in our situation.

Case 1: $\varphi(\bar{x})=\varphi(\overline{0})$ for all $x \in \mathbb{R}^{d}$.

For given $K \in \mathcal{K}_{0}^{d}$, choose points $x_{1}, \ldots, x_{d+1}$ with $K \subset \bar{x}_{1} \vee \cdots \vee \bar{x}_{d+1}=: P$. Then $\varphi(\overline{0}) \subset \varphi(K) \subset \varphi(P)=\varphi\left(\bar{x}_{1}\right) \vee \cdots \vee \varphi\left(\bar{x}_{d+1}\right)=\varphi(\overline{0})$, hence

$$
\varphi(K)=\varphi(\overline{0}) \quad \text { for } K \in \mathcal{K}_{0}^{d} .
$$

Case 2: $\varphi(\bar{x})=\varphi(\overline{0})$ for at least one point $x \neq 0$, but not for all $x \in \mathbb{R}^{d}$.

Let $A:=\left\{y \in \mathbb{R}^{d}: \varphi(\bar{y})=\varphi(\overline{0})\right\}$, then $A$ is convex. Let $x \in A \backslash\{0\}$ and put $y:=x / 2$, then also $y \in A$. Since the convex set $A$ is different from $\mathbb{R}^{d}$, there are points $u, v, w \in \mathbb{R}^{d} \backslash A$ such that $\bar{u} \cap \bar{v}=\overline{0}$ and $w \in(x \vee u) \cap(y \vee v)$. Then $\varphi(\overline{0}) \subset \varphi(\bar{w}) \subset(\varphi(\bar{x}) \vee \varphi(\bar{u})) \cap(\varphi(\bar{y}) \vee \varphi(\bar{v}))=\varphi(\bar{u}) \cap \varphi(\bar{v})=\varphi(\overline{0})$ and hence $\varphi(\bar{w})=\varphi(\overline{0})$, a contradiction.

Gruber's [1] treatment of his Case 2.4 starts with the following proposition (though formulated differently):

(P1) In an n-dimensional affine space, let $M$ be a fixed convex body and let $\mathcal{F}$ be a family of $n$-dimensional convex bodies such that $K \neq M$ for all $K \in \mathcal{F}$ and $K_{1} \cap K_{2}=$ $M$ whenever $K_{1}, K_{2} \in \mathcal{F}$ and $K_{1} \neq K_{2}$. Then $\mathcal{F}$ is at most countable.

In fact, choosing a dense sequence in the space and associating with each set $K \in \mathcal{F}$ the first term of the sequence contained in the set $K \backslash M$ (which has interior points), we 
construct an enumeration of $\mathcal{F}$. We will use Proposition (P1) to exclude the following two cases.

Case 3: $\varphi(\bar{x}) \neq \varphi(\overline{0})$ for all $x \in \mathbb{R}^{d} \backslash\{0\}, \varphi(\overline{0}) \neq \overline{0}$.

Case 4: $\varphi(\bar{x}) \neq \varphi(\overline{0})$ for all $x \in \mathbb{R}^{d} \backslash\{0\}, \varphi(\overline{0})=\overline{0}$; there exists a point $p \in \mathbb{R}^{d}$ with $\operatorname{dim} \varphi(\bar{p}) \geq 2$.

Assume, first, that Case 4 holds with $d=2$. Let $x_{1}, y_{1} \in \mathbb{R}^{2}$ be linearly independent, and choose points $x_{ \pm 1}^{\prime} \in \varphi\left( \pm \bar{x}_{1}\right) \backslash\{0\}, y_{ \pm 1}^{\prime} \in \varphi\left( \pm \bar{y}_{1}\right) \backslash\{0\}$. Let $a, b, c$ be any three of the points $x_{1},-x_{1}, y_{1},-y_{1}$, and let $a^{\prime}, b^{\prime}, c^{\prime}$ be the corresponding points chosen in their $\varphi$-images. Then $(\bar{a} \vee \bar{b}) \cap \bar{c}=\overline{0}$, hence $(\varphi(\bar{a}) \vee \varphi(\bar{b})) \cap \varphi(\bar{c})=\overline{0}$. Thus, $c^{\prime}$ cannot be in the positive hull of $a^{\prime}$ and $b^{\prime}$. Since this holds for all choices of $a, b, c$, the set $\left\{x_{1}^{\prime}, x_{-1}^{\prime}, y_{1}^{\prime}, x_{-1}^{\prime}\right\}$ must be of the form $\{u,-\lambda u, v,-\mu v\}$ with $u, v \in \mathbb{R}^{2} \backslash\{0\}$ and $\lambda, \mu>0$. But then the set $\varphi\left(x_{1}\right)$, for example, cannot be two-dimensional, since otherwise a choice of $x_{1}^{\prime}$ violating the latter condition would be possible. Since we may choose $x_{1}=p$, Case 4 cannot occur for $d=2$.

Now we show (simultaneously) that Case 3 cannot occur and that Case 4 cannot occur if $d \geq 3$. In Case 4 , assume that $d \geq 3$ and let $p$ be as described there; in Case 3 , we set $p=0$.

Put $B:=\varphi(\bar{p})$ and $b:=\operatorname{dim} B$, then $b \geq 1$ in Case 3 and $b \geq 2$ in Case 4 .

By a sheet we understand a set $\{\lambda p+\mu u: \lambda \in \mathbb{R}, \mu>0\}$, where $u$ is a vector linearly independent from $p$ (thus, for $p=0$, a sheet is just a ray without its endpoint $0)$. A sheet is called bad if it contains a point $x$ with $\varphi(\bar{x} \vee \bar{p}) \subset \operatorname{lin} B$. Let $x$ be in a sheet. Then $\varphi(\bar{x}) \cap \varphi(\bar{p})=\varphi(\bar{x} \cap \bar{p})=\varphi(\overline{0})$. If $\varphi(\bar{x} \vee \bar{p})=B$, then $\varphi(\bar{x}) \vee \varphi(\bar{p})=\varphi(\bar{p})$, thus $\varphi(\bar{x}) \subset \varphi(\bar{p})$ and hence $\varphi(\bar{x}) \cap \varphi(\bar{p})=\varphi(\bar{x}) \neq \varphi(\overline{0})$, a contradiction. This shows that $\varphi(\bar{x} \vee \bar{p}) \neq B$. If $x, y$ are in different sheets, then $(\bar{x} \vee \bar{p}) \cap(\bar{y} \vee \bar{p})=\bar{p}$, hence $\varphi(\bar{x} \vee \bar{p}) \cap \varphi(\bar{y} \vee \bar{p})=B$. It follows from (P1) (applied in lin $B$ ) that there are at most countably many bad sheets. The other sheets are called good.

Suppose that $b \geq d-1$. We want to apply $(\mathrm{P} 1)$ in $\mathbb{R}^{d}$, with $M=B$ and with $\mathcal{F}$ defined as follows. Let the set $S$ contain precisely one point from every good sheet, and no other elements, and put $\mathcal{F}:=\{\varphi(\bar{x} \vee \bar{p}): x \in S\}$. If $x \in S$, then $\varphi(\bar{x} \vee \bar{p}) \not \subset \operatorname{lin} B$, hence $\operatorname{dim} \varphi(\bar{x} \vee \bar{p})=d$. Thus the conditions of $(\mathrm{P} 1)$ are satisfied. It follows that $\mathcal{F}$ is countable. This is a contradiction, since there are uncountably many good sheets (here $d \geq 3$ is used for $p \neq 0$ ). This shows that $b \leq d-2$. In particular, $d \geq 3$ also in Case 3 .

Let $k \in\{1, \ldots, d-b\}$. A set $\left\{x_{1}, \ldots, x_{k}\right\}$ of $k$ points in $\mathbb{R}^{d}$, briefly a $k$-set, is called full if $\operatorname{dim} \varphi\left(\bar{x}_{1} \vee \cdots \vee \bar{x}_{k} \vee \bar{p}\right) \geq b+k$. A $k$-flat $E \subset \mathbb{R}^{d}$ is called general if $0 \notin E$ in Case 3, and if $\operatorname{dim} \operatorname{aff}(E \cup \operatorname{lin}\{p\})=k+2$ in Case 4 .

If $x_{1}$ is contained in a good sheet, then $B \subset \varphi\left(\bar{x}_{1} \vee \bar{p}\right) \not \subset \operatorname{lin} B$, hence $\left\{x_{1}\right\}$ is a full 1 -set. We assert the following.

(P2) Let $k \in\{2, \ldots, d-b\}$. In every general $(k-1)$-flat $E \subset \mathbb{R}^{d}$ there is a full $k$-set. 
We prove this by induction with respect to $k$. Let $k=2$. Let $E \subset \mathbb{R}^{d}$ be a general 1-flat. Since $E$ is general, each of its points, with at most countably many exceptions, is contained in a good sheet, and different points of $E$ are in different sheets. Choose a point $x_{1} \in E$ in a good sheet. Then $\left\{x_{1}\right\}$ is a full 1-set. If there exists $y \in E \backslash\left\{x_{1}\right\}$ such that $\left\{x_{1}, y\right\}$ is a full 2-set, we are done. Otherwise, for each $y \in E \backslash\left\{x_{1}\right\}$, the 2-set $\left\{x_{1}, y\right\}$ is not full. This implies that $A:=\operatorname{lin} \varphi\left(\bar{x}_{1} \vee \bar{p}\right)$ is of dimension $b+1$ and that $\varphi(\bar{y} \vee \bar{p}) \subset A$. Since $\varphi\left(\bar{y}_{1} \vee \bar{p}\right) \cap \varphi\left(\bar{y}_{2} \vee \bar{p}\right)=B$ for different $y_{1}, y_{2} \in E \backslash\left\{x_{1}\right\}$, this contradicts $(\mathrm{P} 1)$.

Let $k \in\{2, \ldots, d-b-1\}$ and suppose (P2) has been proved for this number. Let $E \subset \mathbb{R}^{d}$ be a general $k$-flat. Choose a general $(k-1)$-flat $F \subset E$ and a full $k$-set $\left\{x_{1}, \ldots, x_{k}\right\}$ in $F$. If there exists $y \in E \backslash F$ such that $\left\{x_{1}, \ldots, x_{k}, y\right\}$ is a full $(k+1)$-set, we are done. Otherwise, for each $y \in E \backslash F$, the $(k+1)$-set $\left\{x_{1}, \ldots, x_{k}, y\right\}$ is not full. This implies that $A:=\operatorname{lin} \varphi\left(\bar{x}_{1} \vee \cdots \vee \bar{x}_{k} \vee \bar{p}\right)$ is of dimension $b+k$ and that $\varphi(\bar{y}) \subset A$. For any $(k-1)$-flat $F^{\prime} \subset E$ which is parallel to $F$ (but different from it) and hence also general, we choose a full $k$-set $\left\{y_{1}, \ldots, y_{k}\right\}$ in $F^{\prime}$ and put $K_{F^{\prime}}:=\bar{y}_{1} \vee \cdots \vee \bar{y}_{k} \vee \bar{p}$. Then $\varphi\left(K_{F^{\prime}}\right) \subset A$ and $\operatorname{dim} \varphi\left(K_{F^{\prime}}\right)=b+k$. We can choose uncountably many such flats $F^{\prime}$ such that any two of them, say $F_{1}$ and $F_{2}$, satisfy $K_{F_{1}} \cap K_{F_{2}}=\bar{p}$ (observe that $d-b-1 \leq d-2$ in Case 3 and $d-b-1 \leq d-3$ in Case 4$)$, thus $\varphi\left(K_{F_{1}}\right) \cap \varphi\left(K_{F_{2}}\right)=B$. Since $\operatorname{dim} A=b+k,(\mathrm{P} 1)$ yields a contradiction. This completes the induction and thus the proof of (P2).

The case $k=d-b$ yields uncountably many convex bodies $K_{F}$ such that $\operatorname{dim} \varphi\left(K_{F}\right)=d$ and $\varphi\left(K_{F_{1}}\right) \cap \varphi\left(K_{F_{2}}\right)=B$ for $F_{1} \neq F_{2}$. By (P1), this is a contradiction.

Case 5: $\varphi(\overline{0})=\overline{0}$, and $\operatorname{dim} \varphi(\bar{x})=1$ for all $x \in \mathbb{R}^{d} \backslash\{0\}$.

For every $x \in \mathbb{R}^{d} \backslash\{0\}$, the image $\varphi(\bar{x})$ is a nondegenerate segment containing 0 ; let $x^{\prime} \neq 0$ be one of its endpoints (arbitrarily chosen should 0 not be an endpoint). We shall first show that 0 is always one of the endpoints. Let $x_{1}, \ldots, x_{d+1} \in \mathbb{R}^{d}$ be the vertices of a simplex containing 0 in its interior. The set $\left\{x_{1}^{\prime}, \ldots, x_{d+1}^{\prime}, 0\right\}$ has a Radon partition, that is, a decomposition into two subsets whose convex hulls have nonempty intersection.

First case: The only Radon partition is that into the sets $\{0\}$ and $\left\{x_{1}^{\prime}, \ldots, x_{d+1}^{\prime}\right\}$. In that case, $x_{1}^{\prime}, \ldots, x_{d+1}^{\prime}$ are affinely independent and are the vertices of a simplex containing 0 in its interior. If now 0 were not an endpoint of, say, $\varphi\left(\bar{x}_{1}\right)$, then $\varphi\left(\bar{x}_{1}\right) \cap$ $\left(\varphi\left(\bar{x}_{2}\right) \vee \cdots \vee \varphi\left(\bar{x}_{d+1}\right)\right) \neq \overline{0}$, in contradiction to $\bar{x}_{1} \cap\left(\bar{x}_{2} \vee \cdots \vee \bar{x}_{d+1}\right)=\overline{0}$.

Second case: There exists a different Radon partition, say

$$
\operatorname{conv}\left\{x_{1}^{\prime}, \ldots, x_{m}^{\prime}\right\} \cap \operatorname{conv}\left\{x_{m+1}^{\prime}, \ldots, x_{d+1}^{\prime}, 0\right\} \neq \emptyset
$$

without loss of generality, where $m \in\{1, \ldots, d\}$. The only possibility for the intersection is the set $\{0\}$, since

$$
\left(\bar{x}_{1} \vee \cdots \vee \bar{x}_{m}\right) \cap\left(\bar{x}_{m+1} \vee \cdots \vee \bar{x}_{d+1}\right)=\overline{0} .
$$

It follows that $0 \in \operatorname{conv}\left\{x_{1}^{\prime}, \ldots, x_{m}^{\prime}\right\}$. Therefore, 0 is contained in the relative interior of the convex hull of less than $d+1$ affinely independent points among $x_{1}^{\prime}, \ldots, x_{d+1}^{\prime}$, 
say $0 \in \operatorname{relint} \operatorname{conv}\left\{x_{1}^{\prime}, \ldots, x_{k}^{\prime}\right\}$ with $2 \leq k \leq d$. As in the first case, each image $\varphi\left(\bar{x}_{i}\right)$, $i \in\{1, \ldots, k\}$, is the segment with endpoints $x_{i}^{\prime}$ and 0 . Let $a:=\left(x_{1}+\cdots+x_{k}\right) / k$. Then $\bar{a} \subset \bigvee_{i=1}^{k} \bar{x}_{i}$ and $\bar{a} \cap \bigvee_{i \neq j} \bar{x}_{i}=\overline{0}$ for $j=1, \ldots, k$, hence $\varphi(\bar{a}) \subset \bigvee_{i=1}^{k} \varphi\left(\bar{x}_{i}\right)$ and $\varphi(\bar{a}) \cap \bigvee_{i \neq j} \varphi\left(\bar{x}_{i}\right)=\overline{0}$ for $j=1, \ldots, k$. Since $\varphi(\bar{a}) \neq \overline{0}$, this is impossible.

We have proved that, for each $x \in \mathbb{R}^{d}$, the image $\varphi(\bar{x})$ is a segment with endpoints 0 and $x^{\prime}$; thus we can define a mapping $f: \mathbb{R}^{d} \rightarrow \mathbb{R}^{d}$ by $f(x):=x^{\prime}$. It satisfies $f(0)=0$. The next steps serve to show, finally, that $f$ is a linear map.

In the following, we continue to denote $f(x)$ by $x^{\prime}$, and we write $\tilde{x}:=0 \vee x^{\prime}$; thus $\varphi(\bar{x})=\tilde{x}$. By $R_{x}:=\{\lambda x: \lambda>0\}$ we denote the ray (without its endpoint) spanned by $x \neq 0$. Rays $R_{x}, R_{y}$ are called opposite if $R_{x}=-R_{y}$. Vectors $x, y \neq 0$ are called opposite if their spanned rays are opposite. For vectors $x, y \neq 0$ we write $y \succ x$ and $x \prec y$ if $y=\lambda x$ with $\lambda>1$.

First we show that $f$ maps rays into subsets of rays. Let $x \in \mathbb{R}^{d} \backslash\{0\}$, then $x^{\prime} \neq 0$. Let $y \in R_{x}$. If $x \succ y$, then $\varphi(\bar{y}) \subset \varphi(\bar{x})=\tilde{x}$, hence $y^{\prime} \in R_{x^{\prime}}$. If $y \succ x$, then $\tilde{x} \subset \tilde{y}$, hence again $y^{\prime} \in R_{x^{\prime}}$. Thus, $f\left(R_{x}\right) \subset R_{f(x)}$. If $\bar{x} \cap \bar{y}=\overline{0}$, then $\tilde{x} \cap \tilde{y}=\overline{0}$, hence different rays are mapped into different rays.

Let $S \subset \mathbb{R}^{d}$ be a two-dimensional linear subspace. Let $x, y \in S$ be linearly independent. Then $\bar{x} \cap \bar{y}=\overline{0}$, hence $\tilde{x} \cap \tilde{y}=\overline{0}$. If $y^{\prime}$ is opposite to $x^{\prime}$, we choose $z \in S$ linearly independent from $x$ and with $\bar{y} \cap \bar{z}=\overline{0}$. Then $z^{\prime}$ cannot be opposite to $x^{\prime}$, since otherwise $\tilde{z} \cap \tilde{y} \neq \overline{0}$. Hence, we can assume without loss of generality that $x^{\prime}$ and $y^{\prime}$ are linearly independent. Let $S^{\prime}$ be the subspace spanned by $x^{\prime}$ and $y^{\prime}$. Then $f\left(R_{x}\right), f\left(R_{y}\right) \subset S^{\prime}$. If $a, b \in S, z \in a \vee b$ and $a^{\prime}, b^{\prime} \in S^{\prime}$, then $\bar{z} \subset \bar{a} \vee \bar{b}$, hence $\tilde{z} \subset \tilde{a} \vee \tilde{b} \subset S^{\prime}$ and thus $z^{\prime} \in S^{\prime}$. This yields, first, that $z^{\prime} \in S^{\prime}$ for $z \in \operatorname{pos}\{x, y\}$. Let $z \in \operatorname{pos}\{x,-y\} \backslash R_{-y}$. Choose $a \in R_{x}$ and $b \in R_{y}$ with $a \in z \vee b$. Then $\tilde{a} \subset \tilde{z} \vee \tilde{b}$. Since $\tilde{a} \neq \overline{0}$, this is only possible if $z^{\prime} \in S^{\prime}$. Similarly, each $z \in \operatorname{pos}\{y,-x\} \backslash R_{-x}$ is mapped into $S^{\prime}$. For each of the remaining points $z \in S$ we can choose points $a, b \in S$ with $z \in a \vee b$ and $a^{\prime}, b^{\prime} \in S^{\prime}$, hence $z^{\prime} \in S^{\prime}$. We have proved that $f(S) \subset S^{\prime}$, thus the image of any two-dimensional subspace under $f$ is contained in a two-dimensional subspace.

With the same notations as before, suppose the vector $(-x)^{\prime}$ were opposite to $y^{\prime}$. Choose points $a \in R_{-x}$ and $b \in R_{y}$ and set $c:=(a+b) / 2$. Then $\tilde{a} \subset R_{(-x)^{\prime}}$ and $\tilde{b} \subset R_{y^{\prime}}$. From $\bar{c} \subset \bar{a} \vee \bar{b}$ and $\bar{c} \cap \bar{a}=\bar{c} \cap \bar{b}=\overline{0}$ it follows that $\tilde{c} \subset \tilde{a} \vee \tilde{b}$ and $\tilde{c} \cap \tilde{a}=\tilde{c} \cap \tilde{b}=\overline{0}$, a contradiction. Now the argument used above in the treatment of Case 4 for $d=2$ shows that the two pairs of opposite vectors $x,-x, y,-y$ must be mapped under $f$ into two pairs of opposite vectors and that, necessarily, $(-x)^{\prime}$ is opposite to $x^{\prime}$ and $(-y)^{\prime}$ is opposite to $y^{\prime}$.

Let $x$ and $z$ be opposite vectors. Choose a two-dimensional linear subspace $S$ containing them. As shown, $x^{\prime}$ and $(-x)^{\prime}$ are opposite, and $z^{\prime} \in R_{(-x)^{\prime}}$. Hence, the images $x^{\prime}$ and $z^{\prime}$ are on a line through 0 . If $x, z, 0$ are on a line, in this order, then $\tilde{z} \subset \tilde{x}$, hence $x^{\prime}, z^{\prime}$ and 0 are on a line. Now it follows that the images of any three points on a line through 0 are on a line.

We want to show that $f$ maps points on an arbitrary line to points on a line. It suffices to do this for points in a two-dimensional linear subspace, so we restrict ourselves to the subspace $S$ introduced above. To see that the images of three collinear points on a line not passing through zero are collinear, is more difficult, for the reason 
that there seems to be no direct easy way of showing that the restriction of $f$ to a ray is injective.

A point $x$ is called regular if there exists a point $y \succ x$ with $f(y) \neq f(x)$. Let $x, y, z$ be three different points that are, in this order, on a line not passing through 0 . Suppose that $y$ is a regular point. Choose $w \succ y$ with $f(w) \neq f(y)$. Suppose that $f(y)$ were not on the line through $f(x)$ and $f(z)$. From $\bar{y} \subset \bar{x} \vee \bar{z}$ we have $\tilde{y} \subset \tilde{x} \vee \tilde{z}$. Moreover, $\bar{w} \cap(\bar{x} \vee \bar{z})=\bar{y}$ implies $\tilde{w} \cap(\tilde{x} \vee \tilde{z})=\tilde{y}$ and hence $\tilde{w}=\tilde{y}$, a contradiction. Thus, we have proved:

(P3) If the points $x, y, z$ are, in this order, on a line not passing through 0 and if $y$ is regular, then the images $f(x), f(y), f(z)$ are on a line.

Next we show that any ray contains regular points arbitrarily close to 0 .

(P4) For any $x \neq 0$ there exists $y$ with $y \prec x$ and $f(y) \neq f(x)$.

If this is false for some $x \neq 0$, then $f(y)=f(x)$ for all $y \prec x$. By the definition of the map $\varphi$,

$$
\varphi(\overline{0})=\bigcap_{i} \psi^{*}\left(K_{i}\right)
$$

for any sequence $\left(K_{i}\right)_{i}$ in $\mathcal{K}_{(0)}^{d}$ with $0 \in \operatorname{int} K_{i}$ and $K_{i} \downarrow \overline{0}$. We choose the sequence so that $K_{i+1} \subset \operatorname{int} K_{i}$ for all $i$; then $\varphi\left(K_{i+1}\right) \subset \psi^{*}\left(K_{i}\right)$ (see (11) below). For each $i$, there is $y_{i} \in K_{i+1}$ with $f\left(y_{i}\right)=f(x)$. It follows that $f(x) \in \varphi\left(0 \vee y_{i}\right) \subset \varphi\left(K_{i+1}\right) \subset \psi^{*}\left(K_{i}\right)$ and hence that $f(x) \in \varphi(\overline{0})$. Since $\varphi(\overline{0})=\overline{0}$ and $f(x) \neq \overline{0}$, this is a contradiction. This proves $(\mathrm{P} 4)$.

Now we can prove that any $x \neq 0$ is regular. Choose a regular point $y$ independent of $x$ and let $z:=2 y-x$, then $z \neq 0$. Define $z_{0}:=\lambda_{0} z$ with

$$
\lambda_{0}:=\inf \{\lambda>0: f(\lambda z)=f(z)\} .
$$

Then $z_{0} \neq 0$, according to $(\mathrm{P} 4)$. First case: $f\left(z_{0}\right)=f(z)$. Let $w:=z_{0}$ and let $y_{0} \in \bar{y} \cap(x \vee w)$. Choose any $x_{0} \succ x$, and let $w_{0} \in \bar{w} \cap$ aff $\left\{x_{0}, y_{0}\right\}$. From the definition of $z_{0}$ it follows that $f\left(w_{0}\right) \neq f(z)=f(w)$. Second case: $f\left(z_{0}\right) \neq f(z)$. Then let $w_{0}:=z_{0}$. By the definition of $z_{0}$, any point $w$ with $w_{0} \prec w \prec z$ satisfies $f(w)=f(z) \neq f\left(w_{0}\right)$. We may choose such a point $w$ so that for $y_{0} \in \bar{y} \cap(x \vee w)$, the line aff $\left\{w_{0}, y_{0}\right\}$ intersects the ray $R_{x}$ in some point $x_{0} \succ x$. Now in both cases we have that $y_{0}$ is regular (since $y_{0} \in \bar{y}$ ), hence by (P3) the images $f(w), f\left(y_{0}\right), f(x)$ are collinear, and $f\left(w_{0}\right), f\left(y_{0}\right), f\left(x_{0}\right)$ are collinear. Since $f\left(w_{0}\right) \neq f(w)$, we get $f\left(x_{0}\right) \neq f(x)$; thus $x$ is regular.

In view of (P3), this proves that $f$ maps collinear points into collinear points.

To prove that $f$ is injective, we only need to consider $x \neq x_{0}$ with $x \prec x_{0}$. Choose $y_{0} \prec y$ independent of $x$ with $f\left(y_{0}\right) \neq f(y)$, and let $z$ be the intersection point of $x \vee y$ and $x_{0} \vee y_{0}$. Since $f(x), f(z), f(y)$ are collinear and $f\left(x_{0}\right), f(z), f\left(y_{0}\right)$ are collinear, we have $f(x) \neq f\left(x_{0}\right)$.

We can finally identify $f$. We have seen that $f$ is injective, and maps collinear points into collinear points. Its image is not contained in a line, because it maps different 
rays into different rays. Therefore, as in [1], $f$ is a non-singular affine transformation. Since $f(0)=0$, we have $f \in G L(d)$.

It remains to show that $\varphi(K)=f(K)$ for $K \in \mathcal{K}_{0}^{d}$. If $x \in K \in \mathcal{K}_{0}^{d}$, then $\tilde{x}=$ $\varphi(\bar{x}) \subset \varphi(K)$, hence $f(K) \in \varphi(K)$. Suppose that $x \notin K$. Then $K \cap \bar{x}=\bar{z}$ with $z=\lambda x$ and $0 \leq \lambda<1$. This gives $\varphi(K) \cap \tilde{x}=\tilde{z}=(\lambda x)^{\sim}=\lambda \tilde{x}$ (since $f$ is a linear map), hence $f(x) \notin K$, since $f(x) \neq 0$. Altogether this shows that $\varphi(K)=f(K)$ for $K \in \mathcal{K}_{0}^{d}$ and finishes the consideration of Case 5 .

Concluding, the only possible cases are those where either

$$
\varphi(K)=B \quad \text { for } K \in \mathcal{K}_{0}^{d}
$$

with $B:=\varphi(\overline{0})$, or

$$
\varphi(K)=g K \quad \text { for } K \in \mathcal{K}_{0}^{d},
$$

with a fixed linear transformation $g \in G L(d)$.

We show now that these are also the only possibilities for the original map $\psi^{*}$. Let $K^{\prime}, K \in \mathcal{K}_{0}^{d}$ with $K^{\prime} \subset \operatorname{int} K$. In the defining relation

$$
\varphi\left(K^{\prime}\right)=\bigcap_{i} \psi^{*}\left(K_{i}\right), \quad K_{i} \downarrow K^{\prime}, K^{\prime} \subset \operatorname{int} K_{i},
$$

we may choose $K$ for one of the $K_{i}$, hence

$$
K^{\prime} \subset \operatorname{int} K \Rightarrow \varphi\left(K^{\prime}\right) \subset \psi^{*}(K)
$$

Let $C$ be a convex body with $\varphi(K) \subset \operatorname{int} C$. We have $\varphi(K)=\bigcap_{i} \psi^{*}\left(K_{i}\right)$ for any sequence $\left(K_{i}\right)_{i}$ in $\mathcal{K}_{(0)}^{d}$ with $K_{i} \downarrow K$ and $K \subset \operatorname{int} K_{i}$. Since $\psi^{*}\left(K_{i}\right) \subset C$ for sufficiently large $i$ and $\psi^{*}(K) \subset \psi^{*}\left(K_{i}\right)$, we conclude that

$$
\varphi(K) \subset \operatorname{int} C \Rightarrow \psi^{*}(K) \subset C .
$$

Assume, first, that $\varphi(K)=B$ for $K \in \mathcal{K}_{0}^{d}$. Let $K \in \mathcal{K}_{(0)}^{d}$. Choose a convex $C$ body with $B \subset \operatorname{int} C$. Then (11) with $K^{\prime}=\overline{0}$ and (12) give $B \subset \psi^{*}(K) \subset C$. Since this holds for all $C$ with $B \subset \operatorname{int} C$, we conclude that $\psi^{*}(K)=B$.

Now let $\varphi(K)=g K$ for $K \in \mathcal{K}_{0}^{d}$, with a fixed $g \in G L(d)$. Let $K \in \mathcal{K}_{(0)}^{d}$, and choose $K^{\prime}, K^{\prime \prime} \in \mathcal{K}_{(0)}^{d}$ such that

$$
K^{\prime} \subset \operatorname{int} K, \quad K \subset \operatorname{int} K^{\prime \prime} .
$$

Then $\varphi(K)=g K \subset \operatorname{int} g K^{\prime \prime}$, and (11) and (12) give $g K^{\prime} \subset \psi^{*}(K) \subset g K^{\prime \prime}$. Since this together with $g K^{\prime} \subset g K \subset g K^{\prime \prime}$ holds for all $K^{\prime}, K^{\prime \prime}$ satisfying (13), we conclude that $\psi^{*}(K)=g K$.

Finally, since $\psi^{*}(K)=\psi(K)^{*}$ for $K \in \mathcal{K}_{(0)}^{d}$, in case (9) we have $B \in \mathcal{K}_{(0)}^{d}$ and then $\psi(K)=B^{*}$ for all $K \in \mathcal{K}_{(0)}^{d}$. In case $(10), \psi(K)=g^{-t} K^{*}$ for $K \in \mathcal{K}_{(0)}^{d}$. This completes the proof of the theorem. 


\section{References}

[1] Gruber, P., The endomorphisms of the lattice of convex bodies. Abh. Math. Sem. Univ. Hamburg 61 (1991), 121-130.

[2] Gruber, P., The endomorphisms of the lattice of norms in finite dimensions. Abh. Math. Sem. Univ. Hamburg 62 (1992), 179-189.

[3] Ludwig, M., Intersection bodies and valuations. Amer. J. Math. 128 (2006), 1409-1428.

[4] SchneIder, R., Convex Bodies - the Brunn-Minkowski Theory. Cambridge University Press, Cambridge 1993.

Károly J. Böröczky

Alfréd Rényi Institute of Mathematics

Hungarian Academy of Sciences

Reáltanoda u. 13-15

H-1053 Budapest, Hungary

carlos@renyi.hu

Rolf Schneider

Mathematisches Institut

Albert-Ludwigs-Universität

Eckerstr. 1

D-79104 Freiburg i. Br., Germany

rolf.schneider@math.uni-freiburg.de 